\title{
Retorno a Karlsruhe: una experiencia de investigación con la Tabla Periódica para aprender la estructura y propiedades de los elementos químicos
}

\author{
Jordi Domènech-Casal \\ Institut Marta Estrada, Granollers (Barcelona). Departament de Didàctica de les Matemàtiques i les \\ Ciències Experimentals, Universitat Autònoma de Barcelona.jdomen44@xtec.cat \\ ORCID: https:/ / orcid.org/0000-0002-7324-0000
}

[Recibido: 28 Febrero 2018. Revisado: 11 Junio 2018. Aceptado: 25 Agosto 2018]

Resumen: La estructura atómica y la Tabla Periódica son elementos clave en la enseñanza de las ciencias, por constituir un enlace entre la Física y la Química, y por el proceso histórico de su construcción científica. Se describe una experiencia de Aprendizaje Basado en Proyectos en la que el alumnado emula el proceso de construcción de la Tabla Periódica a partir de varios ciclos de indagación. La actividad se cierra con la celebración de un congreso científico escolar, en el cual el alumnado debe realizar predicciones de las características físicas y propiedades químicas de distintos elementos. Se analizan los aprendizajes y visiones del alumnado sobre la secuencia, y se valoran en relación al desarrollo de la Competencia Científica.

Palabras clave: Tabla Periódica, Método de Estudio de Caso, Aprendizaje Basado en Proyectos, Competencia Científica

Back to Karlsruhe: a research experience on the Periodic Table to learn on structure and properties of chemical elements

\begin{abstract}
Atomic structure and the Periodic Table are key elements in Science Education, as they constitute a bridge between Physics an Chemistry, and because of the epistemic value of the historic process of their construction. We describe a Project-Based Learning sequence where students reproduce the inductive process to construct the Periodic Table through several inquiry cycles. The activity ends up with the celebration of a scholar scientific congress where students make predictions on the physical and chemical properties of several elements. We analize the learning outomes and students perspectives on the sequence in the frame of the development of the scientific competence.
\end{abstract}

Keywords: Periodic Table, Case-Studies Method, Project-Based Learning, Scientific Competence

Para citar este artículo: Domènech-Casal, J. (2019) Retorno a Karlsruhe: una experiencia de investigación con la Tabla Periódica para aprender la estructura y propiedades de los elementos químicos. Revista Eureka sobre Enseñanza y Divulgación de las Ciencias 16(1), 1201. doi:10.25267/Rev_Eureka_ensen_divulg_cienc.2019.v16.11.1201

\section{Introducción}

La Tabla Periódica constituye un punto clave en el desarrollo de la química como ciencia y su relación con la física (Scerri 2007). Llegar a ella fue un hito. En particular, porque hasta mediados del siglo XIX coexistían todavía distintas formas de usar conceptos como valencia, peso atómico y peso molecular, además de las confusiones entre molécula, substancia y elemento (Cid 2009). El congreso de Karlsruhe (1860) sirvió para sentar las bases para un léxico y conceptos comunes en la química, como la adopción de las masas atómicas, los acuerdos en la representación de compuestos químicos, el concepto de valencia, y, especialmente, la distinción entre molécula y átomo. Una vez establecido el concepto de átomo y elemento químico, en la búsqueda de un orden fundamental que diera sentido a las distintas características, se generaron distintas formas de ordenarlos, con niveles crecientes de sofisticación. El químico Döberieiner (1829) estableció similitudes entre distintos elementos y variaciones regulares de sus masas y los agrupó según sus propiedades químicas, formando lo

\author{
Revista Eureka sobre Enseñanza y Divulgación de las Ciencias \\ Universidad de Cádiæ. APAC-Eureka. ISSN: 1697-011X \\ http:/ / dx.doi.org/10.25267/Rev_Eureka_ensen_divulg_cienc.2019.v16.i1.1201 \\ http:/ / reuredcuca.es
}


que se llamaron Tríadas: grupos de tres elementos químicos de propiedades similares (CloroBromo-Yodo, Calcio-Estroncio-Bario, Azufre-Selenio-Telurio...) que mantenían proporciones regulares entre sus masas. Tiempo después, otro investigador, Chancourtois (1862), propuso ordenar los elementos según su masa atómica en sucesivas vueltas de hélice, observando que cada siete elementos se reproducían parecidas propiedades químicas. Otro investigador, Newlands (1864), ordena los elementos en columnas verticales de siete elementos cada una, observando la misma repetición (al llegar al octavo elemento se reproducían similares propiedades químicas), en lo que llamó la Ley de las Octavas. Posteriormente Mendéleev (1869) analizó las relaciones peso atómico/volumen atómico y las asoció a las propiedades físicas y las valencias de los elementos, distribuyendo los elementos en forma de tabla. Observando la periodicidad de las características y espacios vacíos en la tabla, pudo predecir la existencia de tres elementos todavía no descubiertos, que serían similares al Aluminio, el Silicio y el Bor, a los que llamó Eka-Aluminio, Eka-Silicio y Eka-Bor. Tiempo después se descubrieron esos nuevos elementos: el Galio, el Escandio, y el Germanio, que confirmaron las propiedades físico-químicas que Mendéleev predijo para ellos. Una historia que a día de hoy lleva a una tabla que consta de más de 100 elementos y continúa creciendo: a finales de 2015 se descubrieron 4 nuevos elementos químicos, el Nihonio (Nh), Moscovio (Mc), Tenesina (Ts) y el último elemento, Oganesón (Og). Éste último es el número 118, completando el séptimo período de la tabla habitado por inestables elementos superpesados, que acerca la química a la teórica isla de estabilidad de elementos superpesados que se produciría a partir del elemento 120. Si bien esta narración parece azarosa, y la Tabla Periódica un constructo singular, lo cierto es que hasta seis investigadores distintos llegaron a propuestas parecidas a la de Mendéleev de forma independiente (Scerri 2007). La Tabla Periódica y su atronadora lógica, eran, al parecer, inevitables, y son para cualquier científico una hermosa representación de la conexión entre la estructura de los elementos y su comportamiento químico. Por ello, no sólo es una referencia obligada como metáfora de la creación del conocimiento científico, sino un escenario privilegiado para la enseñanza de las ciencias, si bien con algunos obstáculos que describimos a continuación.

\section{Marco Teórico}

\section{La enseñanza de la Tabla Periódica}

En la enseñanza de la química, la Tabla Periódica tiene una doble vertiente. Por un lado, la vertiente epistémica: el proceso científico e histórico de inducción a partir de la observación de pautas, la discusión en el marco de la comunidad científica y su utilidad para realizar predicciones de propiedades de elementos todavía no descubiertos es un ejemplo paradigmático del proceso de creación del conocimiento científico. Por otro lado, es también una sistemática para el aprendizaje de distintos conceptos vinculados al comportamiento de los elementos químicos (Ben-Zvi y Gemut, 1998), permitiendo ilustrar relaciones entre los niveles submicroscópicos de la estructura del átomo y los comportamientos químicos de los elementos (Linares 2004, 2005). Franco-Mariscal y Oliva (2013a) concretan que el profesorado de secundaria, en particular, identifica como aspectos claves en la enseñanza de la Tabla Periódica:

a) Propiedades físicas de los elementos y propiedades químicas de los compuestos (en especial conocer algunos elementos y sus propiedades)

b) Regularidad, orden y periodicidad;

c) Interpretación de la Tabla Periódica en función de la estructura atómica; 
d) Presencia de los elementos químicos en la vida diaria;

e) Revisión histórica de la clasificación de los elementos;

f) Naturaleza de la ciencia, aspectos metodológicos y axiológicos.

Varios autores describen que el alumnado, al acabar $4^{\circ}$ de ESO (15-16 años), presenta deficiencias en la comprensión de las propiedades que diferencian los elementos químicos, dificultades que persisten al acabar $2^{\circ}$ de bachillerato (17-18 años) (Cokelez y Dumon 2005, García-Carmona 2006, Franco-Mariscal y Oliva 2013b) y lo atribuyen a la metodología de enseñanza. En el aprendizaje de la Tabla Periódica, los alumnos de secundaria suelen encontrar problemas en desarrollar un interés sobre el tema, aspectos relacionados con la memorización, falta de conceptos previos como el concepto de átomo, dificultades en la comprensión de magnitudes (confusión entre volumen o la masa o dificultades con conceptos abstractos como electronegatividad), la percepción de la periodicidad y su utilidad y la coexistencia de datos de distintos niveles organizativos (macroscópico -substancia elemental- y submicroscópico -átomo-) (Franco-Mariscal y Oliva-Martínez 2012). En este aspecto, participan también las concepciones del alumnado en relación a la estructura del átomo, que, como describen varios autores (Cokelez y Dumon 2005), suelen presentar distintos niveles de sofisticación, no siempre suficientes y en ocasiones representativos de distintas etapas del proceso histórico de construcción del modelo de átomo (Griffiths y Preston 1992).

Las aproximaciones al trabajo con la Tabla Periódica suelen partir de un trabajo previo con la estructura del átomo y presentarla como apoyo para la nomenclatura química y posteriormente las configuraciones electrónicas, ignorando los principios y procesos históricos que llevaron a su construcción (Franco-Mariscal y Oliva 2013b, Martínez y Quijano 2010). La Tabla se usa entonces como un soporte para la síntesis de datos y propiedades con los que abordar conceptos como el enlace químico o el concepto de valencia. Existen algunas experiencias que centran el esfuerzo didáctico en la propia Tabla, mediante juegos del estilo de rompecabezas o cartas que usan las regularidades y periodicidades de la tabla como mecánicas de juego (Kavak 2012, Franco-Mariscal, Oliva-Martínez y Bernal-Márquez 2012, Joag 2014, Franco-Mariscal, Oliva-Martínez, Blanco-López y España-Ramos 2016). Algunos de estos juegos se orientan a los aspectos estructurales de la tabla de forma deductiva, de modo que el alumnado, partiendo de un conocimiento previo de la lógica de la Tabla, coloque los elementos en su lugar correcto. A pesar del interés epistemológico del proceso inductivo de construcción de la Tabla Periódica, es difícil encontrar en la bibliografía descripciones de aplicaciones exitosas de experiencias orientadas a recrear el proceso de inducción que llevó a su confección. La comprensión de la Tabla Periódica y su importancia es un ejercicio didáctico complejo (Crisswell 2007) y requiere elaborar etapas de exploración, reflexión y aplicación. En particular, algunos autores consideran que la expectativa de que los alumnos por sí mismos podrán inducir los patrones es optimista, y se requieren andamios (Larson, Long y Briggs 2012) que promuevan, por ejemplo, un progreso en el establecimiento de patrones, en primer lugar de carácter local (con pocos elementos) y luego más generales. Así mismo, se ha sugerido el uso de analogías, como la comparación de la Tabla Periódica con un calendario mensual (Oliva 2010), que podríamos resumir así: en el pasar de los días, el octavo día vuelve a tener características similares al inicial (vuelve a ser lunes), hay distintos tipos de días con características propias (los lunes son distintos a los jueves) y algún tipo de día es especialmente distinto (los gases nobles y los domingos tienen, definitivamente, características muy especiales). Algunas propiedades (el cansancio y la electronegatividad) progresarían de manera gradual a lo largo de la Tabla y el calendario, y ambos permiten hacer predicciones de cómo será el próximo lunes o metal alcalino. 


\section{La Indagación com práctica científica}

La enseñanza de las ciencias persigue el dominio de modelos científicos, las habilidades de razonamiento y estrategias de construcción y validación del conocimiento científico (Hodson 1994, Pedrinacci et al 2012, Jiménez-Aleixandre 2011). PISA propone estructurar esta definición de competencia científica en tres dimensiones (OCDE 2016, Garrido y Simarro 2015): conceptual, procedimental y epistémica. La dimensión conceptual comprende el dominio de conceptos, modelos científicos y su aplicación a contextos reales. La dimensión procedimental incluye las prácticas y habilidades de razonamiento científico en la que se basa la investigación empírica. La dimensión epistémica trata el marco lógico y social de validación del conocimiento en el que se desarrollan las preguntas y teorías, y la naturaleza de la ciencia. El desarrollo de estas dimensiones requiere metodologías activas en las que el alumnado participe en primera persona en la creación de conocimiento científico en contextos relevantes y problematizados (Hodson 1994, Gilbert 2006, Sanmartí, Burgoa y Nuño 2011, Blanco, España y Rodríguez 2012).

\section{La Enseñanza de las Ciencias Basada en la Indagación}

La enseñanza de las ciencias basada en la indagación (ECBI, IBSE, en sus siglas en inglés) ha sido propuesta como una metodología útil en este sentido (Simarro, Couso y Pintó 2013). La ECBI propone estructurar la enseñanza haciendo que el alumnado participe en primera persona en actividades investigativas dirigidas al desarrollo de los conceptos científicos (Osborne y Dillon 2008, Caamaño 2012, Llewellyn 2005, Rocard 2007). En esta metodología el alumnado parte de fenómenos para formular preguntas investigables, priorizar la observación, sacar conclusiones de datos, formular explicaciones basadas en pruebas y conectar la explicación con otros conocimientos científicos. La participación en estos procesos sería una vía para el desarrollo de la dimensión conceptual (los conceptos construidos por el alumnado), procedimental (las habilidades investigativas) y epistémica (la argumentación y las dinámicas sociales de creación del conocimiento científico). Algunos autores describen que es complejo que esas expectativas se cumplan, porque es difícil construir a partir de pequeñas investigaciones escolares conceptos científicos a un nivel y complejidad suficiente como para formar un marco global (Viennot 2011). Otros autores añaden que en su aplicación se han desarrollado versiones muy rudimentarias del proceso de indagación, pobres en eventos de modelización y argumentación (Couso 2014, 2015, Hattie 2009). Se ha propuesto que un análisis de los fenómenos más progresivo, que ofrezca distintas miradas a un mismo fenómeno, y acompañe mediante la argumentación la construcción de modelos explicativos cada vez más sofisticados (Modelización Basada en la Indagación) constituiría una vía de interés para el desarrollo de la competencia científica (Windshitl, Thompson y Braten 2008, Berland et al 2016).

\section{Los estudios de caso: un ABP como práctica investigadora}

Formulado como metodología por Kilpatrick (1918), el ABP ha cobrado un nuevo interés como vía de trabajo competencial (Larmer, Mergendoller y Boss 2015). Incluye distintos tipos, que comparten la existencia de un propósito externo en la actividad de los alumnos (construir un producto, resolver un problema,...) que instrumentaliza el aprendizaje. A diferencia de las Unidades Didácticas convencionales, en las que suele existir un contexto final al que se transfieren los conceptos y procedimientos, en las actividades ABP el contexto de transferencia supone el primer contacto del alumnado con la actividad (Domènech-Casal -en edición-) y se considera que el aprendizaje de conceptos y habilidades se produce en su transferencia de forma continua al contexto. Varios autores inciden en que el ABP puede constituir una vía para el desarrollo de la competencia científica en las ciencias (Sanmartí 
2016, Sanmartí y Márquez 2017) siendo necesario estudiar con atención aspectos como la conexión de los conceptos científicos con el propósito del proyecto y la inclusión de actividades de síntesis o sistematización (Domènech-Casal -en edición-).

El Método de los Estudios de Caso se inscribe como tipo particular de Aprendizaje Basado en Proyectos (ABP) que comparte con la ECBI el desarrollo activo de aspectos procedimentales y epistémicos. En los Estudios de Caso se propone al alumnado un escenario inicial con pruebas a interpretar para que dé sentido al escenario o resolver el problema que se plantea mediante el uso instrumental de conceptos científicos, la discusión y la argumentación (Christensen y Hansen 1986, Wasserman 1999). Es un método de aplicación en todas las materias, usado habitualmente en la enseñanza de ciencias sociales (derecho, economía,...), ciencias de la salud e ingeniería, como vía para un desarrollo más profundo de conocimientos y procedimientos (Hutchings 1993, Herreid 1994, Hudson y Buckley 2004). En esta metodología, el alumnado no dirige su actividad a construir un concepto científico, sino que se le proporcionan pruebas y se le explican los conceptos científicos de forma paulatina, para que sean construidos por el alumno en su transferencia a la resolución del escenario (Cliff y Wright 1996, Cliff y Nesbit-Curtin 2000, Glew 2003, Herreid 2003). Este planteamiento metodológico se puede aplicar a cualquier temática, y puede evitar la formulación parcial e inconexa de conceptos que algunos autores detectan en la aplicación real de la ECBI (Hattie 2009, Viennot 2011).

Los procesos históricos de Creación del conocimiento científico (génesis de la Teoría de la Evolución, o la Tectónica de Placas) y prácticas científicas aplicadas al mundo real (como la genética forense) constituyen escenarios de Estudio de Caso apropiados para el desarrollo de las dimensiones conceptual, procedimental y epistémica, y consideramos que su uso puede ser de utilidad para la comprensión de la Tabla periódica por parte del alumnado. El proceso epistémico de construcción de la Tabla Periódica, con sus distintas etapas (Tríadas de Döberieiner, Octavas de Newlands y Tabla de Mendéleev) se adapta al esquema de un Estudio de Caso y puede ser usado como esquema para el aprendizaje investigativo de la Tabla Periódica.

\section{Objetivos}

Los objetivos de este trabajo son:

-Implementar en el aula de $3^{\circ}$ de ESO una unidad didáctica de aprendizaje de la Tabla Periódica y la Estructura atómica según la metodología de Estudio de Caso y testar aprendizajes de conceptos y habilidades sobre la Tabla Periódica y la estructura atómica.

-Detectar dificultades del alumnado en conceptos clave de la Tabla Periódica y compararlas con las recogidas en la literatura.

-Analizar percepciones de aprendizaje del alumnado al participar en una secuencia diseñada según el método de Estudio de Caso.

-Analizar representaciones del alumnado sobre el átomo. 


\section{Metodología y análisis de datos}

\section{Descripción de la secuencia}

La secuencia se inicia sin haber trabajado previamente con el alumnado ningún contenido de química en el curso. Se proporcionaron a los alumnos, organizados en equipos, cuatro tarjetas, cada una de ellas representando un elemento químico real distinto, con un nombre ficticio, para evitar que búsquedas por internet interfieran en la actividad (Figura 1). A diferencia de otras propuestas de trabajo inductivo con la Tabla periódica (Calatayud, Carbonell, Carrascosa, Furió, Gil, Grima, Hernández, Martínez, Payás, Ribó, Solbes y Vilches, 1990), las tarjetas propuestas incluyen datos relativos a la estructura electrónica y apoyo gráfico que facilita el acceso y establecimiento de relaciones.

Se proponen al alumnado tres retos, que deben llevar a cabo mediante investigación, comparando las tarjetas entre sí: 1) descubrir qué significa cada una de las informaciones que aparecen en las tarjetas; 2) encontrar un modo lógico de ordenar

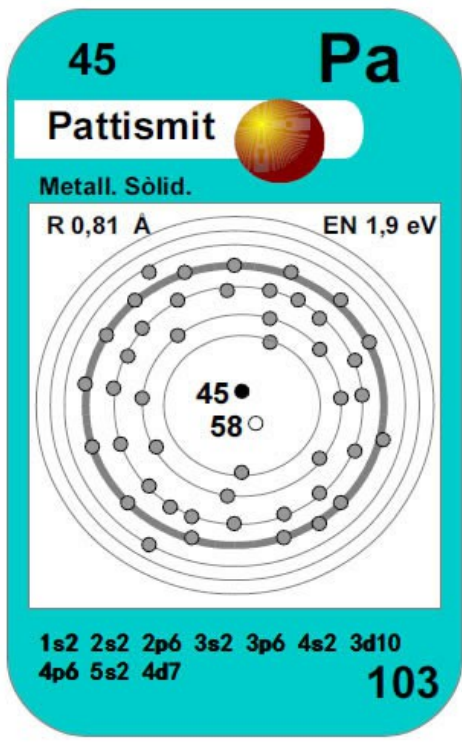

Figura 1. Ejemplo de una de las tarjetas que se proporcionan al alumnado en la actividad. las tarjetas; 3) descubrir qué tarjetas faltan y predecir qué informaciones deberían llevar. A lo largo de varias sesiones de investigación, los estudiantes trabajan en equipos de 3 alumnos, comparando tarjetas y discutiendo entre sí, e incorporando otras tarjetas de elementos que se proporcionan al alumnado paulatinamente, hasta llegar a 56 tarjetas.

Estas sesiones de investigación están intercaladas con sesiones de explicaciones y ejercicios (Figura 2). En las sesiones de explicaciones y ejercicios el profesor explica en base a los elementos químicos reales los conceptos, resuelve como muestra algunos ejercicios clásicos de representación de los conceptos (representación de Lewis, distribución electrónica, formulación química...) y el alumnado realiza los ejercicios, sin una vinculación explícita con las etapas de investigación. Esto se hace para que sean los alumnos los que transfirieran los conceptos y habilidades a la resolución del Estudio de Caso. En cada etapa el alumnado debe ofrecer una solución provisional a cada uno de los tres retos. Cada etapa de investigación tiene una duración de 30-45 min, y las etapas de explicación y ejercicios tienen una duración de 1 hora, excepto la etapa 11, de 3 horas de duración.

Las tarjetas han sido creadas ad hoc para la actividad, y cumplen distintas condiciones:

1) Las que se aportan en las primeras etapas agrupan elementos que siguen el proceso histórico y facilitan la identificación de patrones locales: 1) tarjetas que permiten formar tríadas; 2) tarjetas que permiten formar octavas; 3) tarjetas que completan las "esquinas" de la Tabla Periódica. 
2) A todos los equipos les faltan al terminar 3 tarjetas para completar los períodos del 1 al 5 de la Tabla Periódica. Estas tarjetas son distintas para cada equipo.

3) En las tarjetas iniciales se ha eliminado algún dato (representación del átomo, masa atómica, número atómico), que puede completarse interpretando el significado de otras informaciones de las mismas tarjetas.

4) Algunas tarjetas representan iones $O$ isótopos de elementos.

El propósito de la secuencia es que el alumnado construya los distintos conceptos y habilidades en su instrumentalización para la resolución del problema propuesto. Como conclusión de la secuencia, el alumnado participa en un congreso científico escolar dentro del aula, en el que presenta su propuesta de ordenación y realiza predicciones de las propiedades físico-químicas de los tres elementos que faltan. En conjunto, la secuencia pretende generar espacios para el despliegue de las dimensiones conceptual, procedimental y epistémica de la competencia científica. Los materiales didácticos (tarjetas y ejercicios del alumno) están disponibles para su descarga en: https://bit.ly/2KYUu19

\section{Aplicación de la secuencia}

La secuencia se enmarca en el currículum del bloque 2 ("La materia") de la asignatura de Física y Química de $3^{\circ}$ de ESO en la que el currículo ubica el trabajo con la estructura del átomo y la Tabla Periódica (MECD 2015). Se aplicó durante el curso 2017-2018 en el instituto de secundaria Institut Marta Estrada, de Granollers (Barcelona). En ella participaron un total de 30 alumnos de 14-15 años (13 alumnas y 17 alumnos), con gran heterogeneidad académica. Los alumnos habían, en su mayor parte, participado en cursos anteriores en actividades con metodologías activas (ECBI, ABP) (Llorente, Domènech, Ruiz, Selga, Serra, Domènech-Casal 2017, Domènech-Casal, Gasco, Royo y Vilches 2018) y estaban habituados al trabajo en equipo basado en retos y las dinámicas de auto y coevaluación.

\section{Recogida y análisis de datos}

A lo largo de la aplicación de la secuencia, el docente mantuvo un diario de aula en el que anotó el desarrollo de la implementación. Además, se dispusieron distintos instrumentos:

1) Desarrollo de conceptos y habilidades sobre la Tabla Periódica y la estructura atómica: 
El alumnado realizó una prueba escrita final (disponible para su descarga en https://bit.ly/2KYUu19 ) que incluía preguntas destinadas a evaluar distintos aspectos: a) el dominio del léxico específico, b) Deducir la estructura atómica a partir de datos, c) Ubicar elementos en la Tabla Periódica a partir de sus características, d) Deducir el comportamiento químico y valencias a partir de la estructura atómica de un elemento, e) Formular compuestos, f) Usar la posición en la tabla para predecir distintas características estructurales y comportamientos químicos (carácter metálico, electronegatividad, radio,...). Los resultados se calificaron sobre 10 para cada ítem, y se analizó mediante representación gráfica el nivel medio de éxito del alumnado en cada uno de los ítems. Se compararon los resultados de los distintos ítems para detectar dificultades del alumnado.

2) Encuesta de valoración del alumnado

Al final de la secuencia se propuso al alumnado una encuesta (disponible para su descarga en https://bit.ly/2KYUu19) de valoración de la secuencia y aprendizajes realizados. Se propusieron al alumnado distintas frases sobre su percepción de los aprendizajes y las herramientas y estrategias usadas para que expresaran su grado de apoyo mediante una escala Likert (1->6). Para las distintas frases, se sintetizaron los resultados como porcentaje de alumnos a favor (1-3) y en contra (4-6) y se representaron mediante gráficos de barras.

3) Representaciones del átomo

Al final de la prueba escrita, se pidió al alumnado participante que representara gráficamente un átomo tal como lo imaginaba. Se analizaron las producciones del alumnado y se agruparon según tipos, inicialmente según las propuestas por otros autores (Cokelez y Dumon 2005), que se han ido adaptando hasta acoger todas las producciones.

Por razones accidentales, se dispone de los datos de los exámenes de 29 alumnos/as y de las encuestas y representaciones de átomos de 28 alumnos/as.

\section{Resultados}

\section{Aplicación de la secuencia}

$\mathrm{Al}$ recibir las primeras tarjetas, los alumnos identificaron rápidamente las figuras centrales de cada tarjeta como átomos. Después de la primera sesión de explicaciones y ejercicios sobre estructura del átomo, todos los equipos fueron capaces de identificar en las tarjetas los elementos correspondientes a número atómico, número másico, neutrones, protones y electrones y completar las informaciones al respecto que faltaban en algunas de las tarjetas. En la siguiente ronda de tarjetas (etapa 4), 8 de los 10 equipos identificaron -sin ser instruidos específicamente para ello- tríadas de elementos y en la etapa 6 los 10 equipos habían ya identificado alguna tríada, y la mitad de ellos alguna octava, basándose principalmente en la

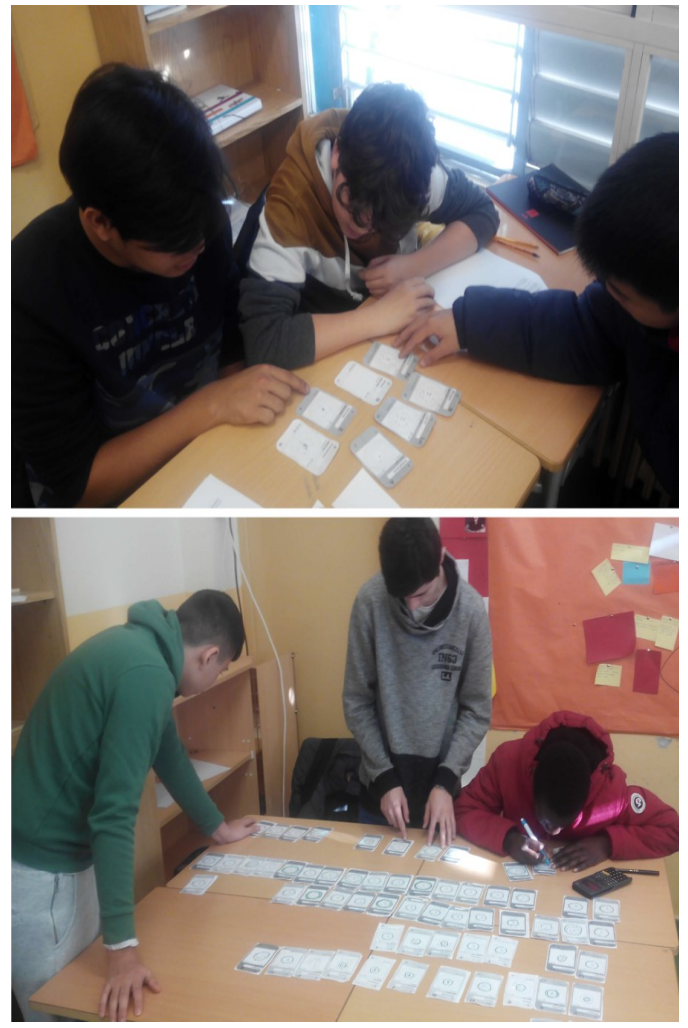

Figura 3. Dos etapas del proceso investigador en las que el alumnado identifica primero tríadas y luego octavas para terminar induciendo toda la Tabla Periódica. 
última órbita completa de los distintos elementos, marcada de manera más intensa en las tarjetas.

En este punto de la actividad todos los equipos asumieron ya el número atómico como elemento principal de la serie, identificando el radio como una variable asociada. Hasta la etapa 8 el alumnado no consiguió dar sentido a las indicaciones sobre distribución electrónica, que permitieron consolidar la estructura en forma de tabla.

La aparición de los metales de transición resultó problemática para todos los equipos. Algunos equipos decidieron "amontonar" las tarjetas representando a los metales de transición sobre los elementos correspondientes del segundo grupo (s2), y otros equipos necesitaron ayuda para ubicar estos elementos.

El avance en la resolución de un sistema de ordenación se aceleró en las últimas etapas. Si bien se esperaban distintas formas de ordenar (en vertical, círculo, horizontal...) todos los equipos terminaron desarrollando Tablas en el formato horizontal (Figura 3). Más que una casualidad, varios alumnos comentaron que al ir avanzando en la actividad conectaron el proyecto con la forma horizontal que recordaban haber visto en lo que llamaron una tabla de átomos del laboratorio.

Completada la resolución, tal como se había previsto, a cada equipo le faltaban en su tabla 3 o 4 tarjetas que se visualizaban como espacios vacíos en la Tabla construida. El encargo final consistía en que cada equipo debía participar en el congreso escolar final con un póster, presentando su predicción de qué elementos no tenían, pero podían predecir que podían existir. En particular, debían definir cómo llamarían a los elementos que faltaban, y qué características predecían que tendrían (radio, electronegatividad, carácter metálico-no metálico, distribución electrónica, valencia,...).

La mitad de los equipos desarrollaron esta parte tal como se esperaba (descubriendo nuevos elementos sobre la Tabla creada por ellos mismos con elementos ficticios) mientras la otra mitad, espontáneamente, prefirió identificar sobre una Tabla Periódica real los elementos que se correspondían con los que faltaban en su tabla. Durante el congreso (Figura 4), se llevó a cabo una actividad de coevaluación en la que los distintos equipos se evaluaban entre sí por su capacidad de justificar sus conclusiones en relación a la ubicación de los elementos desconocidos en la tabla. En las conversaciones durante el congreso se detectó una buena comprensión del papel del número atómico,

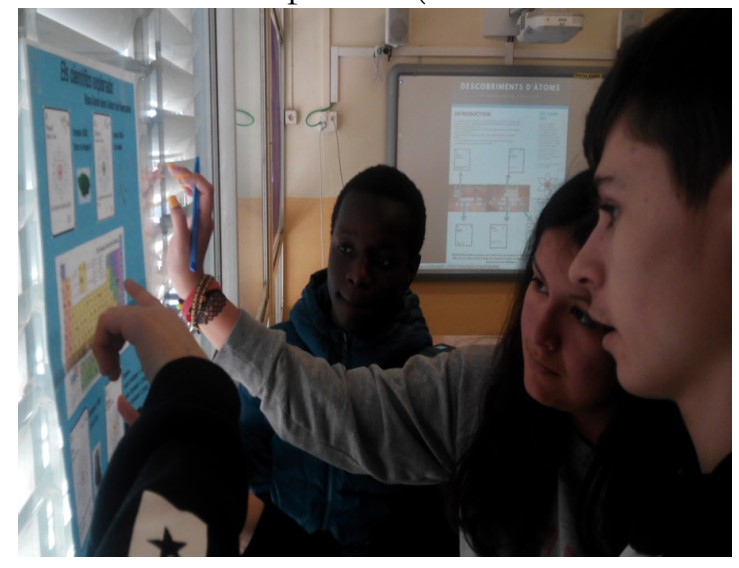

Figura 4. Imagen del congreso final de la actividad, en la que los alumnos justifican sus predicciones. electronegatividad y valencia en relación con la configuración electrónica y la posición en la tabla.

En lo que refiere a las posibilidades de la Metodología de Estudio de Caso para el desarrollo de la Competencia Científica, observamos que la secuencia planteada ha permitido desplegar el trabajo en las tres dimensiones de la competencia científica: dimensión conceptual (concepto y estructura del átomo átomo, enlace, valencia, compuesto), dimensión procedimental (razonamiento inductivo para comprender cómo se rigen las informaciones de las tarjetas o construir una forma de ordenarlas, razonamiento deductivo para realizar predicciones) y epistémica (dinámicas de creación del conocimiento científico mediante argumentación y 
comunicación, construcción paulatina de modelos explicativos provisionales a partir de evidencias parciales).

\section{Habilidades y conocimientos}

Los resultado de la prueba escrita indican que los alumnos desarrollaron principalmente la capacidad de establecer relaciones entre la estructura atómica de un elemento, su representación y su comportamiento químico (Figura 5). También la capacidad de ubicar elementos en la Tabla Periódica obtuvo un desarrollo importante. En cambio, aspectos como el dominio del léxico o la capacidad de formular compuestos (ambas habilidades que no se hallan en el núcleo del problema a resolver del Estudio de Caso) resultaron en menor desarrollo.

En lo referente a aspectos deductivos y conocimiento de las magnitudes que operan a lo largo de la Tabla Periódica, el alumnado fue al final de la secuencia capaz de deducir el carácter metálico o tipo de enlaces partiendo de una ubicación en la Tabla Periódica y, en

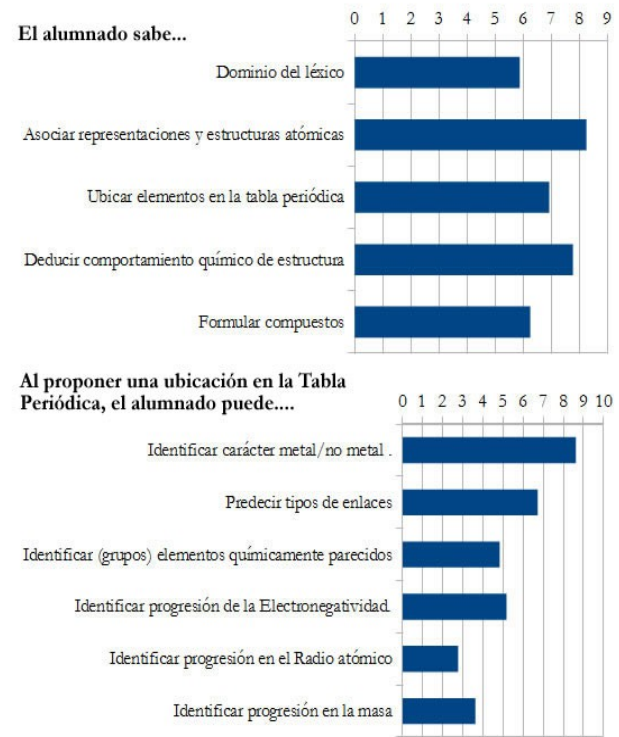
menor grado, la electronegatividad. En

cambio, la progresión del radio o la masa de los elementos a lo largo de la Tabla Periódica o la semejanza química entre elementos de un mismo grupo resulta en valores bajos.

\section{Encuesta de valoración}

Los alumnos consideran que la actividad ha desarrollado todas las instancias propuestas: en la encuesta realizada (Figura 6) consideran que han investigado, discutido, argumentado, comunicado, aprendido química y deducido (más de 4,5 de valoración media). Curiosamente, el eje de la actividad (el pensamiento Inductivo) es el que recibe una puntuación media más baja, de 4,4 .

En lo relativo a los aprendizajes, el alumnado

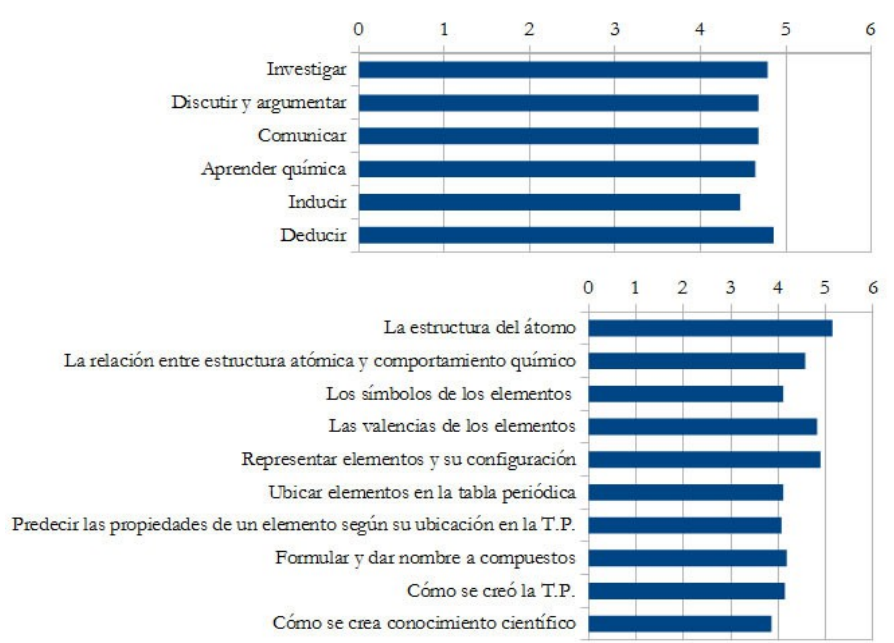

Figura 6. Resultados de la encuesta realizada al alumnado sobre los procesos y aprendizajes vinculados a la actividad realizada. considera (Figura 6) que ha aprendido principalmente la estructura del átomo, el concepto de valencia de un elemento, la representación de elementos y su configuración y la relación entre estructura atómica y comportamiento químico. Algunos elementos clave en el diseño de la actividad (cómo se crea 
conocimiento científico, predecir las propiedades de un elemento según su ubicación en la T.P.) reciben la valoración más baja (3, 8 y 4). Al ser preguntados sobre distintos aspectos del diseño de la actividad en relación al interés que generan y su utilidad para aprender (Figura 7), el alumnado ofrece de media una valoración bastante alta de los distintos aspectos del diseño (la valoración más baja es la inclusión de palabras clave en los materiales y la realización y corrección de la prueba escrita).

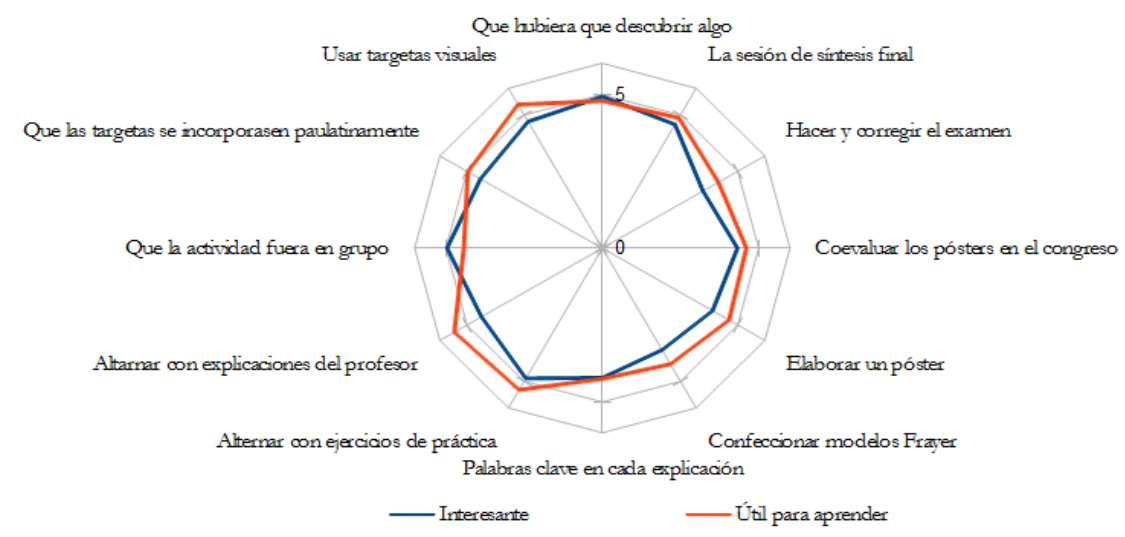

Figura 7. Representación de los valores medios $(1->6)$ del interés o utilidad para el aprendizaje de distintas componentes del diseño didáctico.

En general, el alumnado valora los distintos aspectos del diseño más por su utilidad para aprender que por el interés que generan, con excepción de dos: que la actividad fuera en grupo y que hubiera de descubrir algo. Estos dos elementos del diseño son los únicos en los que el alumnado destaca más su aportación a hacer la actividad interesante que su utilidad para aprender, si bien se valora también alta. Como útiles para el aprendizaje el alumnado valora principalmente que se hayan intercalado explicaciones del profesor, el uso de tarjetas visuales y su incorporación paulatina, la sesión de síntesis final y el hecho de alternar la investigación con ejercicios.

\section{Representaciones del átomo}

Los dibujos de los alumnos se agruparon según similitudes en lo referente al grado de sofisticación en la descripción de los niveles estructurales. De ello resultaron 6 categorías (Tabla 1). En los tres modelos se distinguen variaciones que hacen incidencia especial en los distintos tipos de partículas subatómicas $(\mathrm{B}, \mathrm{D}, \mathrm{F})$ y otros que no inciden en ello (A, C, E). Los modelos más representados han sido los modelos E (8), F (5) y D (7). Los dibujos menos sofisticados son los menos frecuentes, 4 del modelo C, 3 del modelo A y 1 del modelo B.

\section{Discusión y conclusiones}

\section{Aprendizaje de la Tabla Periódica y la Estructura atómica. Metodología de Estudios de Caso}

Como hemos dicho, la secuencia permite el despliegue de las dimensiones conceptual, procedimental y epistémica de la competencia científica. Valoramos que el hecho de proporcionar las tarjetas de los distintos elementos de forma paulatina y siguiendo un orden que promueve el proceso histórico (tríadas, octavas, Tabla) ha sido de utilidad para superar las dificultades descritas por otros autores en el diseño de secuencias de trabajo inductivo para la 
Tabla periódica (Larson, Long y Briggs 2012) y el alumnado completara con éxito el "descubrimiento" de la Tabla.

La intercalación de sesiones de explicación y ejercicios ha constituido para el alumnado un andamio útil. Asimismo, el trabajo iterativo en sucesivos ciclos de investigación con cada nuevo aporte de tarjetas se acerca a la necesidad, propuesta por varios autores, de secuenciar las actividades de indagación como ciclos de elaboración de modelos explicativos cada vez más sofisticados (Windshitl, Thompson y Braten 2008, Couso 2014).

\section{Concepciones y dificultades del alumnado en conceptos clave de la Tabla Periódica}

Las medias obtenidas en la prueba escrita muestran que el alumnado ha adquirido un grado de conocimiento más alto en los aspectos más estrechamente vinculados al reto propuesto (asociar representaciones y estructuras, ubicar elementos y deducir comportamiento químico de estructura). Para los objetivos de aprendizaje menos instrumentalizados para la resolución del reto (domino del léxico y formulación de compuestos) el alumnado ha obtenido resultados aceptables, pero más bajos, lo que es esperable según la filosofía de aprendizaje de los Estudios de Caso. 
Tabla 1. Ejemplos de cada uno de los tipos de representaciones de átomos generados por el alumnado.

\begin{tabular}{|c|c|c|}
\hline Modelos & $\begin{array}{l}\text { Menos sofisticado (sin distinguir } \\
\text { partículas) }\end{array}$ & $\begin{array}{l}\text { Más sofisticado (distinguiendo } \\
\text { partículas) }\end{array}$ \\
\hline $\begin{array}{l}\text { Modelo bloque: se distingue un } \\
\text { solo o como máximo dos niveles } \\
\text { organizativos, el átomo se muestra } \\
\text { como un bloque (Ejemplos E y F). }\end{array}$ & $\begin{array}{l}\text { A: Estructura } \\
\text { simple sin } \\
\text { distinguir } \\
\text { partículas. } 10 \% \\
\text { del alumnado. }\end{array}$ & $\begin{array}{l}\text { B: Estructura } \\
\text { simple } \\
\text { distinguiendo } \\
\text { partículas. } \\
4 \% \text { del } \\
\text { alumnado }\end{array}$ \\
\hline $\begin{array}{l}\text { Modelo estático: se distinguen } \\
\text { más de dos niveles organizativos } \\
\text { (Ejemplos C y D). }\end{array}$ & $\begin{array}{l}\text { C: Dos niveles } \\
\text { organizativos sin } \\
\text { distinguir } \\
\text { partículas. } 15 \% \\
\text { del alumnado. }\end{array}$ & $\begin{array}{l}\text { D: Dos niveles } \\
\text { organizativos } \\
\text { distinguiendo } \\
\text { partículas. } \\
25 \% \text { del } \\
\text { alumnado }\end{array}$ \\
\hline $\begin{array}{l}\text { Modelo dinámico: se distinguen } \\
\text { más de dos niveles organizativos y } \\
\text { se transmite mediante recursos } \\
\text { visuales una visión dinámica del } \\
\text { átomo (Ejemplos A y B). }\end{array}$ & $\begin{array}{l}\text { E: Más de dos } \\
\text { niveles } \\
\text { organizativos sin } \\
\text { distinguir } \\
\text { partículas. 28\% } \\
\text { del alumnado. }\end{array}$ & $\begin{array}{l}\text { F: Más de dos } \\
\text { niveles } \\
\text { organizativos } \\
\text { distinguiendo } \\
\text { partículas. } 18 \% \\
\text { del alumnado. }\end{array}$ \\
\hline
\end{tabular}

$\mathrm{Al}$ analizar qué información es capaz el alumnado de extraer de la ubicación de un elemento en la tabla periódica, en comparación con experiencias descritas por otros autores (Martínez y Quijano 2010), el alumnado ha obtenido resultados bajos en lo relativo a la comprensión del papel de magnitudes como el radio atómico o la masa, si bien obtiene buenos resultados en lo referido a identificar la progresión de la electronegatividad en la Tabla Periódica. Esto contradice lo hallado por otros autores (Larson, Long y Briggs 2012, Franco-Mariscal y OlivaMartínez 2012) en secuencias de aprendizaje sobre la Tabla Periódica, en las que los alumnos identifican fácilmente las relaciones de masa, pero tienen más dificultades en identificar aquéllas vinculadas a la electronegatividad. Esto probablemente se deba al distinto papel que se ha dado a la configuración electrónica al trabajar la Tabla.

Nuestros resultados muestran también que aspectos como la capacidad de identificar el carácter metal/no metal o la predicción de tipos de enlaces de distintos elementos químicos resultan en un nivel alto por parte del alumnado, si bien estas dos capacidades se han trabajado de forma más intensa en las sesiones de explicación-ejercicios que en las sesiones investigativas, y es difícil proponer hasta qué punto la ubicación en un Estudio de Caso habrá sido de utilidad para estos ítems.

\section{Percepción de los aprendizajes por parte del alumnado}

El alumnado valora muy positivamente los aprendizajes logrados, incluyendo aspectos conceptuales y procedimentales, si bien el que menos perciben como aprendido es el objetivo explícitamente epistémico "Cómo se crea conocimiento científico". Las dificultades en el desarrollo y percepción de los objetivos de aprendizaje de naturaleza epistémica han sido descritas en anteriores trabajos con Estudios de Caso (Domènech-Casal 2016) y parecen indicar la necesidad de un trabajo más explícito alrededor de la naturaleza de la ciencia. De igual modo, llama la atención que de distintas habilidades investigativas, todas ellas valoradas por el alumnado como aprendidas, la capacidad de inducir sea la que recibe una valoración 
más moderada, algo llamativo, precisamente en una secuencia donde ha primado mucho el enfoque de trabajo mediante el razonamiento inductivo.

\section{Representaciones del átomo del alumnado}

Las representaciones de átomos elaboradas por el alumnado se corresponden con categorías de representaciones descritas por otros autores para edades similares (Cokelez y Dumon 2005), quienes han observado distintos niveles de sofisticación en ocasiones representativos del proceso histórico de construcción del modelo de átomo (Griffiths y Preston, 1992). Las categorías E, F, y D se corresponden con lo que estos autores denominan Modelo de sistema solar, mientras que la categoría C se corresponde con la de Composición de modelo atómico. Las categorías A y B a lo que estos autores denominan Modelo bola. Estos autores proponen que entre los 16 y 18 años el alumnado suele abandonar paulatinamente el Modelo bola para elegir representaciones del tipo modelo de Lewis, Composición o Sistema Solar. Para 18 años, estos autores muestran que cerca de una tercera parte del alumnado continúa conservando el Modelo bola como representación, mientras que sólo una décima parte del alumnado desarrolla el Modelo Sistema Solar. En nuestro caso (2 años menores), la mayor parte del alumnado ha llegado a desarrollar modelos similares al Modelo Sistema Solar e incluso transmitir de algún modo una componente dinámica. Como otros autores (Griffiths y Preston, 1992) hemos identificado representaciones del átomo que se corresponden con etapas del proceso histórico de construcción del modelo, como por ejemplo el modelo B, que se corresponde con el modelo de Thomson. Ninguno de los alumnos ha elegido usar representaciones de Lewis como representación de un átomo, cosa que parece sugerir que las consideran herramientas analíticas, quizás más vinculadas a otros aspectos, como la deducción del carácter metálico o valencia de los elementos.

Es importante recalcar que la secuencia es el resultado de una transposición didáctica que incluye simplificaciones y analogías, y por ello puede generar concepciones alternativas que conviene prevenir:

-La Tabla Periódica se construyó en base a propiedades de sustancias, y en el proceso no participaron conceptos como la configuración electrónica, pues este aspecto se desarrolló más adelante.

-La secuencia trabaja sólo con los cinco primeros períodos, por lo que debe hacerse un trabajo posterior para transferir los conceptos a otras partes significativas de la tabla, como los Lantánidos o Actínidos o los elementos superpesados de síntesis.

-Aunque no es uno de los ejes de la secuencia, para el trabajo con valencias y su asociación a la estructura electrónica, se han usado las estructuras de Lewis, que otros autores advierten que tienen sus límites (Olivares 2014), pudiendo generar valores de valencias incompletos, como ha ocurrido en la secuencia.

Consideramos que la metodología de Estudio de Caso propuesta es una vía adecuada para la ubicación del aprendizaje en un contexto problematizado y el desarrollo de la Competencia Científica, y a tenor de las valoraciones del alumnado, la existencia de un problema a resolver ha sido uno de los ítems de la secuencia que más interés ha generado y más utilidad ha tenido para el aprendizaje. Esto se ve corroborado por el hecho que los aprendizajes más directamente vinculados a la resolución del reto son los que más se han detectado en la prueba escrita. Es importante destacar que la metodología de Estudios de Caso no sólo permite, sino que además promueve, que el trabajo investigativo se alterne con la introducción y sistematización de contenidos y que estos aspectos (explicaciones del profesor y síntesis final) han sido especialmente valorados por el alumnado en lo relativo a la utilidad para aprender. 
El lector encontrará de interés actividades propuestas por otros autores alrededor de la construcción de la Tabla Periódica con modelos alternativos y dinámicas de gamificación (Franco-Mariscal, Oliva-Martínez y Bernal-Márquez 2012, Anta 2013) o métodos inductivos (Calatayud, Carbonell, Carrascosa, Furió, Gil, Grima, Hernández, Martínez, Payás, Ribó, Solbes y Vilches 1990) y actividades de Estudios de Caso sobre otras temáticas (DomènechCasal 2017). La secuencia forma parte del Itinerario ProjectantCN3 ${ }^{1}$, que reúne distintas secuencias ABP para el despliegue del currículo de Biología y Geología y Física y Química de $3^{\circ}$ de ESO.

\section{Agradecimientos}

Reflexiones incluidas en este artículo se enmarcan en la investigación metodológica del grupo de investigación consolidado LICEC referencia 2014SGR1492) por AGAUR y financiado por el Ministerio de Economía y Competitividad (referencia EDU2015-66643-C2-1-P). El autor agradece la colaboración de profesorado y alumnado del Institut Marta Estrada en la aplicación de las actividades.

\section{Referencias}

Anta A. (2013) Diseña tu propia Tabla Periódica. Educació Química 15, 53-61.

Ben-Zvi N., Gemut S. (1998) Uses and limitations of scientific models: the Periodic Table as an inductive tool. International Journal of Science Education 20(3), 351-360.

Blanco A., España E., Rodríguez F. (2012) Contexto y enseñanza de la competencia científica. Alambique, Didáctica de las Ciencias Experimentales 70, 9-18.

Caamaño A. (2012) ¿Cómo introducir la indagación en el aula? Los trabajos prácticos investigativos. Alambique, Didáctica de las Ciencias Experimentales 70, 83-91.

Calatayud M. L., Carbonell F.,Carrascosa J., Furió C. J., Gil D., Grima J., Hernández J., Martínez J., Payás J., Ribó J., Solbes J., Vilches A. (1990). La construcción de las ciencias físico-químicas. Nau Llibres: Valencia, Spain.

Cid R. (2009) El congreso de Karlsruhe: paso definitivo hacia la química moderna. Revista Eureka sobre Enseñanza y Divulgación de las Ciencias 6(3), 396-407.

Cliff W. H., Nesbitt-Curtin L. (2000) The directed case method. Journal of College Science Teaching 30 (1), 64-66.

Cliff W. H., Wright A. W. (1996) Directed case study method for teaching human anatomy and physiology. Advances in Physiology Education 15, 19-28.

Cokelez, A., Dumon, A. (2005). Atom and molecule: upper secondary school French students' representations in long-term memory. Chemistry Education Research and Practice 6 (3), 119 135.

Couso D. (2014) De la moda de “aprender indagando" a la indagación para modelizar: una reflexión crítica. XXVI Encuentro de Didáctica de las Ciencias Experimentales. Huelva (Andalucía).

Couso D. (2015) La clau de tot plegat: la importancia de "què" ensenyar a l'aula de ciències. Revista Ciències, 29, 29-36.

Crisswell B. (2007) Mistake of Having Students Be Mendeleev for Just a Day. Journal of Chemical Education 84 (7), 1140.

\footnotetext{
${ }^{1}$ Itinerario de proyectos ProjectantCN3 http://sites.google.com/site/projectantcn3/
} 
Chancourtois A.E.B. (1862) Vis tellurique. Comptes rendus de l'Académie des sciences 54 (1862): 757-761, 840-843, 967-971.

Christensen C.R., Hansen A.J. (1986) Teaching and the Case Method. Boston: Harvard Bussiness School Publishing Division.

Döberieiner J. W. (1829) An Attempt to Group Elementary Substances according to Their Analogies. Annalen der Physik und Chemie 20, 301-307.

Domènech-Casal J. (2016) Diseño y caracterización de un Proyecto de Indagación alrededor de la Evolución Humana y la Paleontología. Investigación en la escuela 90, 49-71.

Domènech-Casal J. (2017) Aprendizaje Basado en Proyectos y Competencia Científica. Experiencias y propuestas para el método de Estudios de Caso. X Congreso Enseñanza de las Ciencias Septiembre 2017 (número extraordinario) 5177-5183.

Domènech-Casal, J., Gasco, J., Royo, P., Vilches, S. (2018). CRASH: un proyecto de enseñanza de cinemática y dinámica en el contexto del análisis pericial de accidentes. Revista Eureka sobre Enseñanza y Divulgación de las Ciencias 15 (2), 2103, 1-17.

Domènech-Casal J. (en edición) Contexto y modelo en el Aprendizaje Basado en Proyectos. Apuntes para el ámbito científico. Alambique, Didáctica de las Ciencias Experimentales.

Franco-Mariscal A. J., Oliva-Martínez J. M, Bernal-Márquez S. (2012) Una revisión bibliográfica sobre el papel de los juegos didácticos en el estudio de los elementos químicos. Primera parte: los juegos al servicio del conocimiento de la Tabla Periódica Educación Química 23(3), 338-345.

Franco-Mariscal A. J., Oliva-Martínez J. M. (2012) Dificultades de comprensión de nociones relativas a la clasificación periódica de los elementos químicos: La opinión de profesores e investigadores en educación química. Revista Científica 16(2), 53-71.

Franco-Mariscal A. J., Oliva-Martínez J. M. (2013a) ¿Qué enseñar en secundaria sobre la Tabla Periódica? Educació Química 15, 43-52.

Franco-Mariscal A. J., Oliva J.M. (2013b). Evolución en el alumnado de la idea de elemento químico a lo largo del bachillerato. Revista Eureka sobre Enseñanza y Divulgación de las Ciencias 10(3), 353-376.

Franco-Mariscal A. J., Oliva-Martínez J.M., Blanco-López, A., España-Ramos, E. (2016). A Game-Based Approach To Learning the Idea of Chemical Elements and Their Periodic Classification. J. Chem. Educ., 93 (7), 1173-1190.

García-Carmona A. (2006) La estructura electrónica de los átomos en la escuela secundaria: un estudio de los niveles de comprensión. Educación Química 17(4), 414-423.

Garrido A., Simarro C. (2014) El nou marc d'avaluació de la competència científica PISA 2015: Revisió i reflexions didàctiques. Revista Ciències 28, 21-26.

Gilbert J.K. (2006) On the nature of «context» in chemical education. International Journal of Science Education 28 (9), 957-976.

Glew R. H. (2003) The problem with problem-based medical school education: Promises not kept. Biochemistry and Molecular Biology Education 31, 52-56.

Griffiths A.K., Preston K.R. (1992) Grade-12 students' misconceptions relating to fundamental characteristics of atoms and molecules. Journal of Research in Science Teaching 29, 611-628. 
Hattie J. (2009) Visible Learning: A synthesis of over 800 meta-Analyses relating to achievement. Routledge: New York.

Herreid C. F. (1994) Case studies in science: A novel method for science education. Journal of College Science Teaching 23 (4), 221-229.

Herreid C. F. (2003) The death of problem-based learning? Journal of College Science Teaching 32 (6), 364-66.

Hodson D. (1994) Hacia un enfoque más crítico del trabajo de laboratorio. Enseñanza de las Ciencias 12(3), 299-313.

Hudson J. N., Buckley P. (2004) An evaluation of case-based teaching: Evidence for continuing benefit and realization of aims. Advances in Physiological Education 28, 15-22.

Hutchings P. (1993) Using Cases to improve college teaching: a guide to a more relfective practice. Washington, DC: American Association for Higher Education.

Joag S.D. (2014) An Effective Method of Introducing the Periodic Table as a Crossword Puzzle at the High School Level. Journal of Chemical Education 91 (6), 864-867.

Kavak N. (2012). ChemPoker. Journal of Chemical Education 89 (4), 522-523.

Kilpatrick W.E. (1918) The Project Method: the use of the purposeful act in the educative process. Teachers college, Columbia University.

Larmer J., Mergendoller J., Boss S. (2015) Setting the Standard for Project Based Learning: A Proven Approach to Rigorous Classroom Instruction. Alexandria: ASCD.

Larson K.G., Long G.R., Briggs M.W. (2012) Periodic Properties and Inquiry: Student Mental Models Observed during a Periodic Table Puzzle Activity. Journal of Chemical Education 89, 1491-1498.

Linares R. (2004) Elemento, átomo y sustancia simple. Una reflexión a partir de la enseñanza de la Tabla Periódica en los cursos generales de Química. (Tesis Doctoral). Barcelona: Universidad de Barcelona.

Linares R. (2005) Elemento, átomo y sustancia simple. Diferentes lecturas de la Tabla Periódica. En: Enseñanza de las Ciencias, Núm. Extra VII Congreso Internacional sobre Investigación en Didáctica de las Ciencias.

Llewellyn D. (2005) Teaching High School Science through Inquiry: A case study approach. Corwin Press \& NSTA press.

Llorente I., Domènech X., Ruiz N., Selga I., Serra C., Domènech-Casal J. (2017) Un Congreso Científico en secundaria: articulando el Aprendizaje Basado en Proyectos y la Indagación científica. Investigación en la Escuela 91, 72-89.

Martínez L.D., Quijano M.H. (2010) Propuesta didáctica para la enseñanza y el aprendizaje de la Tabla Periódica desde una perspectiva histórica y epistemológica. II Congreso nacional en Educación en Ciencias y Tecnología (Colombia, 2010).

MECD (2015) Ministerio de Educación, Cultura y Deporte, Real Decreto 1105/2014, de 26 de diciembre, por el que se establece el currículo básico de la Educación Secundaria Obligatoria y del Bachillerato. Boletín Oficial del Estado 2015(3), I, 169.

Mendeleev, D. (1869) On the Relationship of the Properties of the Elements to their Atomic Weights. Zeitschrift für Chemie 12, 405-406.

Newlands J.A.R. (1864) Relations between equivalents. Chemical News 10, 59-60. 
OCDE (2016) PIS A 2015. Draft Science Framework.

Oliva J.M. (2010) Comparando la Tabla Periódica con un calendario: posibles aportaciones de los estudiantes al diálogo de construcción de analogías en el aula. Educació Química 6, $13-22$.

Olivares S. (2014) ¿Formulación química? Nomenclatura química. Revista Eureka sobre Enseñanza y Divulgación de las Ciencias 11(3), 416-425.

Osborne J., Dillon J. (2008) Science Education in Europe: Critical Reflections. Londres: Nuffield Foundation.

Rocard Y. (2007) Science Education Now. Report EU22-845, EU, Brussels.

Sanmartí N. (2016) Trabajo por proyectos: ¿Filosofía o metodología? Cuadernos de Pedagogía $472,44-46$.

Sanmartí N., Márquez C. (2017) Aprendizaje de las ciencias basado en proyectos: del contexto a la acción. Apice, Revista de Educación Cientifica 1 (1), 3-16.

Sanmartí N., Burgoa B., Nuño T. (2011) ¿Por qué el alumnado tiene dificultades para utilizar sus conocimientos escolares en situaciones cotidianas? Alambique, Didáctica de las Ciencias Experimentales 67, 62-68.

Scerri E. (2007). The periodic table. Its Story and its significance. New York: Oxford University Press.

Simarro C., Couso D., Pintó R. (2013) Indagació basada en la modelització: un marc per al treball pràctic. Revista Ciències 25, 35-43.

Viennot L. (2011) Els molts reptes d'un ensenyament de les Ciències basat en la indagació: ens aportaran múltiples beneficis en l'aprenentatge? Revista Ciències 18, 22-36.

Wasserman S. (1999) El estudio de casos como método de enseñanz̧a. Amorroutu Editores: Buenos Aires.

Windshitl M., Thompson J., Braten M. (2008) Beyond the scientific method: model-based inquiry as a new paradigma of preference for school science investigations. Science Education 92, 941-967. 